\title{
Mechanism of enhanced oral absorption of hydrophilic drug incorporated in hydrophobic nanoparticles
}

This article was published in the following Dove Press journal:

International Journal of Nanomedicine

26 July 2013

Number of times this article has been viewed

\section{Liang-Zhong Lv' \\ Chen-Qi Tong' \\ Jia Yu' \\ Min $\mathrm{Han}^{2}$ \\ Jian-Qing $\mathrm{Gao}^{2}$}

'Department of Pharmacy, Zhejiang

Provincial People's Hospital,

Hangzhou, People's Republic of China;

${ }^{2}$ Institute of Pharmaceutics, College

of Pharmaceutical Sciences, Zhejiang

University, Hangzhou, People's

Republic of China
Correspondence: Min Han Institute of Pharmaceutics, College of Pharmaceutical Sciences, Zhejiang University, Hangzhou 310058 , People's Republic of China Email hanmin2@zju.edu.cn

Jian-Qing Gao

Institute of Pharmaceutics, College of Pharmaceutical Sciences, Zhejiang University, Hangzhou 310058, People's Republic of China $\mathrm{Tel} / \mathrm{Fax}+86$ 57I 88208437

Email gaojianqing@zju.edu.cn
Abstract: Hydroxysafflor yellow A (HSYA) is an effective ingredient of the Chinese herb Carthamus tinctorius $L$, which has high water solubility and low oral bioavailability. This research aims to develop a hydrophobic nanoparticle that can enhance the oral absorption of HSYA. Transmission electron microscopy and freeze-fracture replication transmission election microscopy showed that the HSYA nanoparticles have an irregular shape and a narrow size distribution. Zonula occludens 1 protein (ZO-1) labeling showed that the nanoparticles with different dilutions produced an opening in the tight junctions of Caco-2 cells without inducing cytotoxicity to the cells. Both enhanced uptake in Caco-2 cells monolayer and increased bioavailability in rats for HSYA nanoparticles indicated that the formulation could improve bioavailability of HSYA significantly after oral administration both in vitro and in vivo.

Keywords: hydroxysafflor yellow A, nanoparticles, Caco-2 cells, bioavailability, absorption

\section{Introduction}

Improving the high water solubility and low intestinal permeability of Biopharmaceutics Classification System (BCS) Class III drugs has been a focus of study in pharmaceutical research. Generally, BCS Class III drugs are not adapted to oral formulations. Thus, the addition of an absorption enhancer ${ }^{1}$ and chemical modification ${ }^{2}$ is necessary to improve the means of drug delivery ${ }^{3,4}$ and enhance drug bioavailability (BA), respectively. The flower of the safflower plant, Carthamus tinctorius $L$, has been widely used in traditional Chinese medicine for treatment of cerebrovascular and cardiovascular diseases. ${ }^{1}$ Hydroxysafflor yellow A (HSYA) is extracted from the flower of the safflower plant. As the main active component extracted from the safflower plant, it has been demonstrated to have a strong antagonistic effect on the platelet-activating factor receptor ${ }^{5}$ as well as outstanding neuroprotective action in vivo and in vitro. ${ }^{6}$ According to recent studies, HSYA is a BCS Class III $^{1,7}$ hydrophilic drug with low oral bioavailability, which means that it is only administered in clinical therapy via injections. ${ }^{8,9}$ Water-in-oil microemulsion and the self-double-emulsifying drug delivery system have shown great potential in improving the oral absorption of HSYA. ${ }^{10,11}$

In this study, hydrophobic nanoparticle oil solutions were prepared to increase the oral absorption of HSYA. Hydrophobic nanoparticle oil solutions are composed of caprylic/capric triglyceride (GTCC), bean phospholipids, and organic solvents. The nanoparticle oil solutions were formed after the organic solvents were volatilized. Through these procedures, small-sized nanoparticles in a stable, oily environment were prepared, and a formulation that is more stable than emulsions was obtained. 
This study investigated the possible enhancing mechanism of hydrophobic nanoparticles to HSYA.

\section{Material and methods}

\section{Material}

HSYA (98\% purity) was purchased from Chengdu Herb purity Company, Ltd (Chengdu, People's Republic of China), while GTCC (caprylic/capric triglyceride) was obtained from Gattefosse (Saint-Priest, France). Bean phospholipids (Lipoid S 100) were purchased from Lipoid (Ludwigshafen, Germany), and Sephadex G-100 was supplied by the Beijing Investor Science and Technology Development Company, Ltd (Beijing, People's Republic of China). Methyl- $\beta$-cyclodextrin was obtained from the Shanghai Ding Jie Biological Technology Company, Ltd (Shanghai, People's Republic of China). NaN3, amiloride, rhodamine-123, N-acetyl-L-cysteine, polyoxyethylene (10) octylphenyl ether (Triton X-100) and 3-(4,5-dimethylthiazol -2-yl)-2,5-diphenyltetrazolium bromide (MTT) were purchased from Sigma-Aldrich (St Louis, MO, USA). The rabbit anti-ZO-1 antibody was purchased from Abcam (Cambridge, UK), while the FITC-labeled goat anti-rabbit IgG antibody was purchased from Wuhan Boster Biological Engineering Company, Ltd (Wuhan, People's Republic of China). Dulbecco's modified Eagle's medium (DMEM) and fetal bovine serum (FBS) were purchased from Thermo-Fisher Biochemical Products (Beijing, People's Republic of China).

\section{Animals}

Male Sprague-Dawley rats (200 g $\pm 20 \mathrm{~g}$ ) were obtained from the Animal Center of Zhejiang University (Hangzhou, People's Republic of China). The animal experiment was approved by the Animal Ethics Committee of Zhejiang University. The animals were housed in a normal laboratory environment with access to food and water.

\section{Preparation of HSYA-loaded nanoparticles}

Initially, $500 \mathrm{mg}$ of soybean phospholipids were dissolved in $2 \mathrm{~mL}$ methanol. Then, $50 \mathrm{mg}$ of HSYA was added and ultrasonicated to obtain a clarified mixed solution (A). Afterwards, another $500 \mathrm{mg}$ of soybean phospholipids were dissolved in $0.5 \mathrm{~mL}$ ethanol and ultrasonicated, then $10 \mathrm{~mL}$ GTCC was added to obtain a mixture (B). The $\mathrm{A}$ and $\mathrm{B}$ mixtures were mixed and homogenized at 9,500 rpm for 2 minutes (FJ-200 High-Speed Dispersion Homogenizer; Jiangsujintan Jincheng Instruments Co, Jiangsu, People's Republic of China) until a clear and transparent formulation was obtained. The organic solvents were removed by using a rotary evaporator (Shanghaijiapeng Technology Company Ltd, Shanghai, People's Republic of China) for 30 minutes at $40^{\circ} \mathrm{C}$.

\section{Characterization of HSYA-loaded nanoparticles \\ Droplet size and morphology observation}

The newly prepared nanoparticles were filtered through a $0.22 \mu \mathrm{m}$ organic membrane, and the droplet size was measured by using dynamic light scattering (Nano-S 90; Malvern, Worcestershire, UK). The morphology of the nanoparticle was observed through transmission electron microscopy (TEM) (Philips Tecnai 10; Philips, Amsterdam, Netherlands). The particle size, particle size distribution, and aggregation state of the nanoparticles were characterized by freeze-etching (BAF 060; Leica, Germany) replication TEM (FERTEM).

\section{Encapsulation efficiency, drug loading capacity,} and in vitro drug release

The encapsulation efficiency (EE\%) and drug loading capacity (LC\%) were determined by using the Sephadex column method, and calculated using the formulas below. ${ }^{11}$

$$
\begin{gathered}
\mathrm{EE} \%=\frac{\text { HSYA in nanoparticles }}{\text { Total amount of HSYA in dispersion }} \times 100 \% \\
\text { LC } \%=\frac{\text { HSYA in nanoparticles }}{\text { Nanoparticles weight }} \times 100 \%
\end{gathered}
$$

Briefly, $20 \mu \mathrm{L}$ nanoparticles were dissolved in $180 \mu \mathrm{L}$ methanol to release HSYA as the total amount of HSYA. Another $20 \mu \mathrm{L}$ nanoparticles was mixed with water (1:4) to form emulsions, then passed through a Sephadex column. The eluted emulsions from the column were further treated by methanol to release HSYA as the content of HSYA in nanoparticles.

In vitro drug release was performed by using the dialysis bag method. ${ }^{12,13}$ The dialysis bag allowed the transfer of the released drug molecules into the release media while intercepting the nanoparticles. The dialysis bag (molecular weight range: 8,000 to 14,000; Sigma-Aldrich) was boiled for more than 30 minutes and soaked in the release media overnight prior to the experiment; the release media was double-distilled water. One end of the dialysis bag was tied tightly, and $10 \mathrm{~mL}$ release media was placed in the bag. 
The other end of the dialysis bag was closed with a dialysis clip, and the dialysis bag was then placed in a beaker that contained $200 \mathrm{~mL}$ release media. The beaker was placed on a magnetic stirrer (Hangzhou Instrument Electric Co, Ltd) at a rotational speed of $100 \mathrm{rpm}$ at $4{ }^{\circ} \mathrm{C}$. Afterwards, $1 \mathrm{~mL}$ of the freshly prepared HSYA nanoparticles $(3.88 \mathrm{mg} / \mathrm{mL})$ was added to the release media outside the dialysis bag. At the preset time points $(0.5,1,2,4,6,8,12,24,36,48,60$, $72,96,120,144,168$, and 192 hours), $1 \mathrm{~mL}$ of the release sample was withdrawn from the media in the dialysis bag, and equal amounts of fresh release media was immediately added into the dialysis bag to maintain the sink condition. The in vitro release of the HSYA solution was carried out by adding the drug solution to the release media and withdrawing the release sample from the release media in the beaker directly and not from the dialysis bag. All samples were analyzed by high-performance liquid chromatography (HPLC).

\section{Caco- 2 cell culture}

The Caco- 2 cells were cultured in a cell incubator with an atmosphere of $5 \% \mathrm{CO}_{2}$ at $37^{\circ} \mathrm{C}$. The culture medium was DMEM, which contained 4,500 mg/L D-glucose, $584 \mathrm{mg} / \mathrm{L}$ L-glutamine, $3.7 \mathrm{~g} / \mathrm{L} \mathrm{NaHCO} 3$, supplemented with 10\% (volume $[\mathrm{v}] / \mathrm{v}$ ) heat-inactivated FBS, $1 \%(\mathrm{v} / \mathrm{v})$ penicillin, and $1 \%(\mathrm{v} / \mathrm{v})$ streptomycin. The culture medium was replaced every 3 to 4 days. Cell subculturing was performed when the cells reached $80 \%$ to $90 \%$ coverage.

\section{Cell viability assay}

Caco-2 cells were seeded in 96-well plates at a density of 10,000 cells/well and cultured for 24 hours with DMEM culture medium. When the experiment was started, the medium was replaced with $200 \mu \mathrm{L}$ serial dilutions of blank nanoparticles or the control (culture medium). The cells were exposed to the nanoparticles for 2 hours at $37^{\circ} \mathrm{C}$, after which $20 \mu \mathrm{L}$ of MTT reagent $(5 \mathrm{mg} / \mathrm{mL})$ in Phosphate Buffer Solution (PBS) was added to each well, and the mixtures were incubated for 4 hours. Then, $180 \mu \mathrm{L}$ Dimethyl sulfoxide (DMSO) was added to each well, and the mixture was agitated gently to dissolve the crystals completely. The absorbance values were detected by using a KHB ST-360 microplate reader (KHB, Shanghai, People's Republic of China) at $570 \mathrm{~nm}$. Cell viability was calculated as follows:

$$
\text { Cell viability }(\%)=\mathrm{A}_{\text {text }} / \mathrm{A}_{\text {control }} \times 100 \%
$$

\section{Transepithelial electrical resistance measurement in Caco- 2 cells}

Cells were seeded at a density of $2.5 \times 10^{5}$ cells/well in a 12-well polycarbonate membrane transwell (12 mm, $1 \mu \mathrm{m}$ pore size, $0.3 \mathrm{~cm}^{2}$ growth area) obtained from Costar (Silicon Valley, USA). The cells were cultured for 21 days under the previously described culture conditions. The volumes of the culture medium were $0.5 \mathrm{~mL}$ and $1.5 \mathrm{~mL}$ on the apical and basolateral side, respectively. At 21 days, the cell transepithelial electrical resistance (TEER) was measured using Millicell ERS-2 Volt-Ohm Meter (Millipore, Massachusetts, USA) to evaluate cellular integrity, as previously reported. ${ }^{14,15}$ To evaluate the impact of HSYA solutions, HSYA-nanoparticles and blank nanoparticles on cells integrity, $3.88 \mathrm{mg} / \mathrm{mL}$ of HSYA solutions and the same concentration of HSYA-nanoparticles and blank nanoparticles were all diluted 10-fold to incubate with Caco- 2 cells for 2 hours. The TEER was also measured when the formulation was removed after 2 hours of incubation, and the culture was continued up to 24 hours and 48 hours to evaluate if the impact of the formulation on cell integrity was reversible.

\section{Cellular uptake of HSYA}

Cells were seeded at a density of $2.5 \times 10^{5}$ cells/well onto six-well plates and cultured for 14 days for the uptake experiments. Cells were treated with different concentrations of HSYA solution and HSYA nanoparticles for 2 hours. The cells were preincubated with a number of endocytotic inhibitors, such as NaN3 $(1.32 \mathrm{mg} / \mathrm{mL})$, chlorpromazine $(10 \mu \mathrm{g} / \mathrm{mL})$, methyl- $\beta$-cyclodextrin (methyl- $\beta$-CD) $(13.3 \mathrm{mg} / \mathrm{mL})$, and amiloride $(50 \mu \mathrm{M})$, for 30 minutes to understand the endocytotic mechanism of the formulations. Blank nanoparticles were also added to investigate whether they have an effect on Caco-2 cellular uptake of HSYA. At the end of the experiments, the cells were washed three times using ice-cold PBS, then frozen and thawed three times. One $\mathrm{mL}$ of water was added to each well. The wells were probesonicated 25 times to obtain the cell lysates. The lysates were centrifuged at 13,000 rpm for 5 minutes, and the supernatant liquid was analyzed using HPLC.

\section{Cellular transport of HSYA}

Caco-2 cells were seeded onto the 12-well transwell at a density of $2.5 \times 10^{5}$ cells/well and cultured at a condition similar to that during TEER measurements. At 21 days, the transmembrane resistance met the requirements, ${ }^{16,17}$ and the cell model was ready for transport experiments. One $\mathrm{mg} / \mathrm{mL}$ 
of the HSYA solutions was added to the apical side of the cells, and $1.5 \mathrm{~mL}$ DMEM without FBS was added to the basolateral side. At 15, 30, 45, 60, 90, 120, 180, 240, 360, and 480 minutes, $500 \mu \mathrm{L}$ of the sample from the basolateral side was collected and replaced with the same volume of fresh DMEM without FBS. Another group of cells was preincubated with $150 \mu \mathrm{g} / \mathrm{mL}$ of cyclosporin A (CsA, a recognized p-gly-coprotein [p-gp]) for 30 minutes. The cells were preincubated with $0.5 \mathrm{~mL}$ methanol, added to the samples of the nanoparticle group, and then probe-sonicated 25 times $\left(4^{\circ} \mathrm{C}\right.$, $150 \mathrm{~W}$, active every 2 seconds within a 3-second duration). All samples were centrifuged at 13,000 rpm for 5 minutes. Then, $50 \mu \mathrm{L}$ of the supernatant was injected into the liquid chromatography system (Agilent 1,200 system; Agilent, Santa Clara, CA, USA) for investigation.

\section{Effect of HSYA nanoparticles on ZO-I distribution}

The cells were seeded in a 12 -well polycarbonate membrane transwell at a density of $2.5 \times 10^{5}$ cells/well and cultured for 21 days before the experiment, followed by incubation with 10 -fold dilution HSYA nanoparticles for 2 hours at $37^{\circ} \mathrm{C}$. Then, the cells were washed three times with ice-cold PBS and fixed with 4\% paraformaldehyde solution for $30 \mathrm{~min}$ utes, followed by rinsing with ice-cold PBS two times and permeabilization in $0.2 \%$ Triton X-100/PBS for 5 minutes. Non-specific binding was blocked with 5\% Bovine serum albumin (BSA)/PBS for 30 minutes. Primary antibody (rabbit anti-ZO-1 antibody) at a concentration of 1:100 was applied and incubated at room temperature for 2 hours. The cells were washed three times with PBS, and the secondary antibody (FITC-conjugated goat anti-rabbit IgG) was added at a concentration of 1:48 and incubated at room temperature for 1-hour. Finally, the cells were washed three times with PBS and observed under two-photon confocal microscopy (BX61 W1-FV1000; Olympus, Japan).

\section{In vivo pharmacokinetic studies}

Pharmacokinetic studies were carried out by dividing the rats into two groups, with three rats in each group. The animals in the HSYA group were given a dose of $25 \mathrm{mg} / \mathrm{kg}$, which was also the dose given in our previous study. ${ }^{11}$ Another group received the oral HSYA nanoparticles at a dose of $25 \mathrm{mg} / \mathrm{kg}$. After the administration of HSYA solution and HSYA formulations to the rats through oral gavage, blood samples were collected from the eye ground vein into heparinized tubes at preset time points of 5, 15, 30, 45, 60, 90, 120, 180, $240,360,480$, and 1,440 minutes. The blood samples were centrifuged at 4,000 rpm for 10 minutes, and the supernatant was transferred into another plastic tube and stored at $-20^{\circ} \mathrm{C}$ until further analysis.

\section{Determination of HSYA concentrations in plasma}

The plasma concentrations of HSYA were determined through HPLC, as we described in our preliminary work. ${ }^{11}$ Two hundred ul plasma samples were collected in plastic centrifuge tubes with $100 \mu \mathrm{L}$ of $6 \%$ perchloric acid added to precipitate protein. The mixture was vortexed for $2 \mathrm{~min}$ utes. After centrifugation at 13,000 rpm for 10 minutes, the supernatant was detected using HPLC.

\section{HPLC analysis of samples}

Chromatographic conditions were performed according to the literature: ${ }^{18}$ a $_{18}$ column (Agilent SRB- $\mathrm{C}_{18}, 5 \mu \mathrm{m}$, $4.6 \mathrm{~mm} \times 250 \mathrm{~mm}$, Agilent) was used at $40^{\circ} \mathrm{C}$. The mobile phase was $32 \%$ methanol, $2 \%$ acetonitrile, and $66 \%$ phosphate solution $(0.1 \%$ concentration $)$. The injection volume was $50 \mu \mathrm{L}$, and the flow rate was $0.8 \mathrm{~mL} / \mathrm{minute}$. The detection signal was $403 \mathrm{~nm}$.

\section{Pharmacokinetic analysis}

The maximum plasma concentration was defined as $C_{\max }$, while the time to reach $C_{\max }$ was defined as $t_{\max }$. The area under the plasma concentration time curve (AUC) and mean retention time (MRT) were calculated from 0 hours to 12 hours. All parameters were calculated by using Kinetica 4.4 software (Thermo Fisher Scientific Inc., MA, USA).

\section{Statistical analysis}

All values are expressed as mean \pm standard deviation (SD). Statistical significance was assessed using one-way analysis of variance (ANOVA) among more than three groups, with $P<0.05$ considered statistically significant. The means of the two groups were compared by performing two-tailed student's $t$-tests.

\section{Results}

\section{Characterization of HSYA-loaded}

\section{nanoparticles}

Figure $1 \mathrm{~A}$ and $\mathrm{B}$ show that the freshly prepared HSYA nanoparticles were diluted with 10-fold GTCC. The morphology of the diluted nanoparticles was characterized by TEM and FERTEM. Figure 1A shows that the formed nanoparticles exhibited a spherical structure with a relatively uniform size. FERTEM was performed to further characterize the 

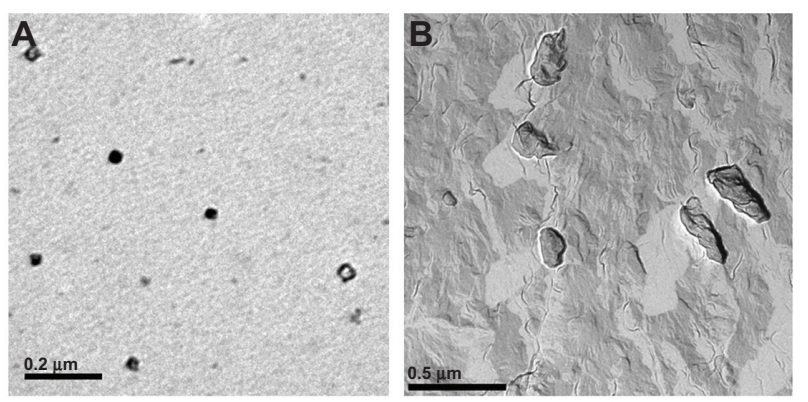

C

Statistics graph (1 measurements)

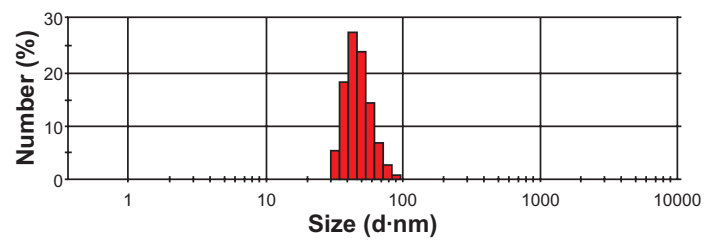

Mean with $+/-1$ standard deviation error bar

Figure I (A) TEM image of HSYA nanoparticles, (B) FERTEM image of HSYA nanoparticles, and (C) Size distribution of freshly prepared HSYA nanoparticles. Abbreviations: FER, freeze-etching replication; HSYA, hydroxysafflor yellow A; TEM, transmission electron microscopy.

surface morphology of the HSYA nanoparticles. The HSYA nanoparticles exhibited irregular morphology. The cross section of the nanoparticles was not flat and had a similar layer- or shell-like structure (Figure 1B). Dynamic light scattering studies (Figure 1C) showed that the mean diameter of the HSYA nanoparticles without dilution was $49.2 \mathrm{~nm} \pm 5.8 \mathrm{~nm}$, and the polydispersity was 0.38 . The encapsulating efficiency and drug loading capacity was $71.68 \%$ and $0.71 \%$, respectively.

The equilibrium dialysis method was used to determine the in vitro release behavior of the HSYA solution and HSYA

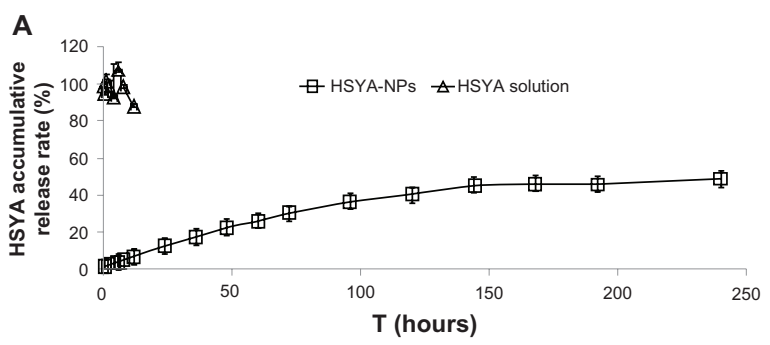

B

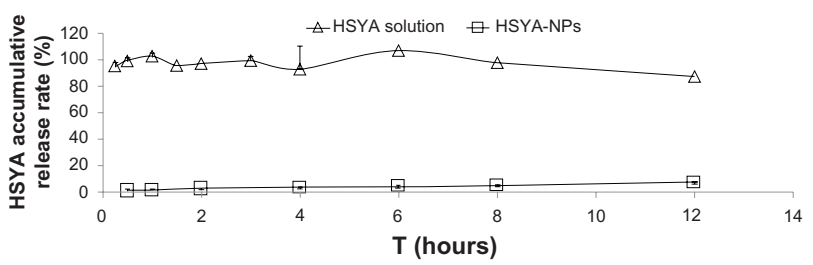

Figure 2 HSYA release from nanoparticles in double-distilled water at $4^{\circ} \mathrm{C}$ $(n=3)$.

Note: Figure 2B is an enlarged version of Figure 2A.

Abbreviations: HSYA, hydroxysafflor yellow A; NP, nanoparticle. nanoparticles, and to evaluate the impact of nanostructures on drug release. In the case of the HSYA solution, complete release was obtained after 0.5 hours, whereas in the case of the HSYA nanoparticles, HSYA was totally released from the nanoparticles after 192 hours (approximately $50 \%$ was totally released), and the release was better sustained in vitro.

\section{Cell viability assay}

Figure 3A shows that the blank nanoparticles diluted 10-, 15-, 30 -, and 50-fold showed almost no cytotoxicity after incubation for 2 hours.

\section{TEER measurement in Caco-2 cells}

Figure 3B shows that after the 2-hour incubation of HSYA solution and HSYA nanoparticles, the TEER of Caco- 2 cells decreased. The TEER did not significantly increase after the HSYA solution or the HSYA-loaded nanoparticles were removed and culture continued for 24 hours and 48 hours. When the blank nanoparticles were incubated for 2 hours, the TEER did not decrease. The TEER increased with continued cell culture. From these results, we hypothesize that HSYA is somewhat toxic to Caco-2 cells, thereby destroying the integrity of the cells. The blank nanoparticles showed almost no effect on the integrity of the cells.

\section{Cellular uptake and transport studies of HSYA}

Figure 4B shows that the apparent permeability coefficient (Papp) of $1 \mathrm{mg} / \mathrm{mL}$ HSYA was $(5.38 \pm 1.58) \times 10^{-7}$, which

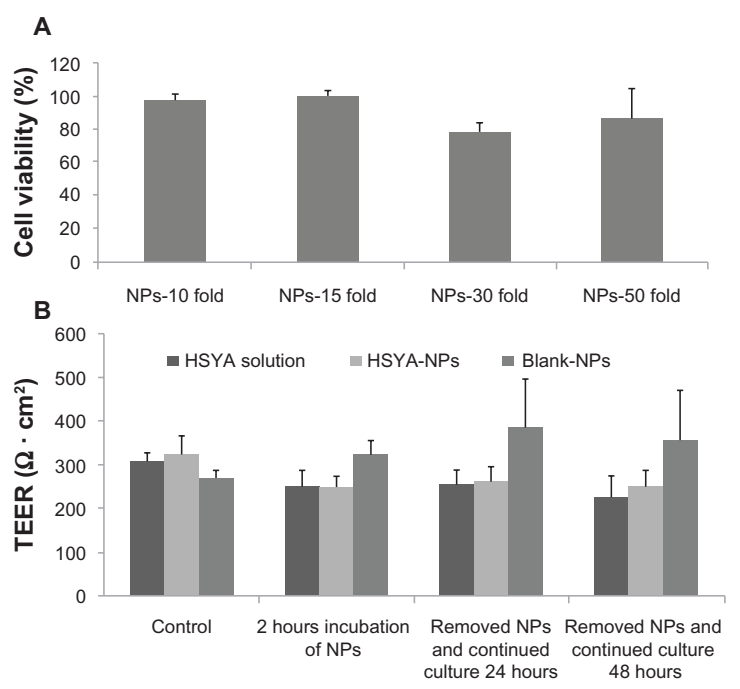

Figure 3 (A) Cytotoxicity of blank nanoparticles on Caco-2 cells after incubation for 2 hours; (B) Cellular integrity studies of incubation with nanoparticles and removal of nanoparticles $(n=3)$.

Abbreviations: HSYA, hydroxysafflor yellow A; NP, nanoparticle; TEER, transepithelial electrical resistance. 
increased to $(1.27 \pm 0.64) \times 10^{-6}$ when the Caco- 2 cells were preincubated with $\mathrm{CsA}$ (p-glycoprotein inhibitors) for 30 minutes. This result indicates the presence of p-glycoprotein efflux, which may be one of the reasons behind poor HSYA absorption. Figure 4A shows that both high and low concentrations of HSYA nanoparticles can significantly promote HSYA absorption.

Endocytotic inhibitor experiments were conducted (Figure 4C) to clarify how the nanoparticles promote HSYA absorption. Prior to the experiments, Caco-2 cells were preincubated for 30 minutes with three kinds of inhibitors, namely, chlorpromazine $(10 \mu \mathrm{g} / \mathrm{mL})$ to inhibit clathrin vesicles, methyl- $\beta-C D(13.3 \mathrm{mg} / \mathrm{mL})$ to inhibit caveolae, and amiloride $(50 \mu \mathrm{M})$ to inhibit pinocytosis. However, the results revealed that the inhibitors did not have any significant effect on HSYA uptake. Blank nanoparticles also have no significant effect on HSYA absorption.

\section{Effect of HSYA-loaded nanoparticles on ZO-I distribution}

$\mathrm{ZO}-1$ is a tight junction-associated protein that is localized on the cytoplasmic surface just beneath the membrane. Figure 5A shows that the ZO-1 of the control group was uniformly distributed in the tight connection region of the cell. However, in comparison to the control cell layers, the individual regions exhibited discontinuous distribution in the HSYA nanoparticle group, and part of the cells exhibited extrusion and deformation.

\section{In vivo pharmacokinetic studies}

The plasma concentration-time profiles of HSYA after intravenous or oral administration of HSYA solution and HSYA nanoparticles to rats are shown in Figure 6, and the pharmacokinetic parameters are summarized in Table 1 . The AUC of HSYA nanoparticles was 23.3-fold greater than that of the HSYA solution.

\section{Discussion}

HSYA is the main component of safflower yellow pigments, which is the aqueous extract of safflower florets. Recent studies investigated the effect of HSYA on lipopolysaccharideinduced inflammatory signal transduction in human alveolar epithelial A549 cells, ${ }^{19}$ which includes inhibition of protein oxidation/nitration, 12/15-lipoxygenase, ${ }^{20}$ exertion of therapeutic actives on cerebral ischemia induced by thrombosis, ${ }^{21}$ and promotion of blood circulation by influencing hemorheology, plasma coagulation, and platelet aggregation, ${ }^{22}$ among others. HSYA is a hydrophilic drug with poor oral bioavail-

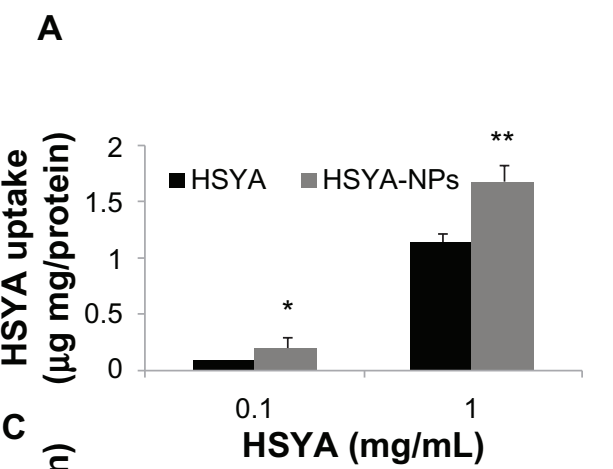

B
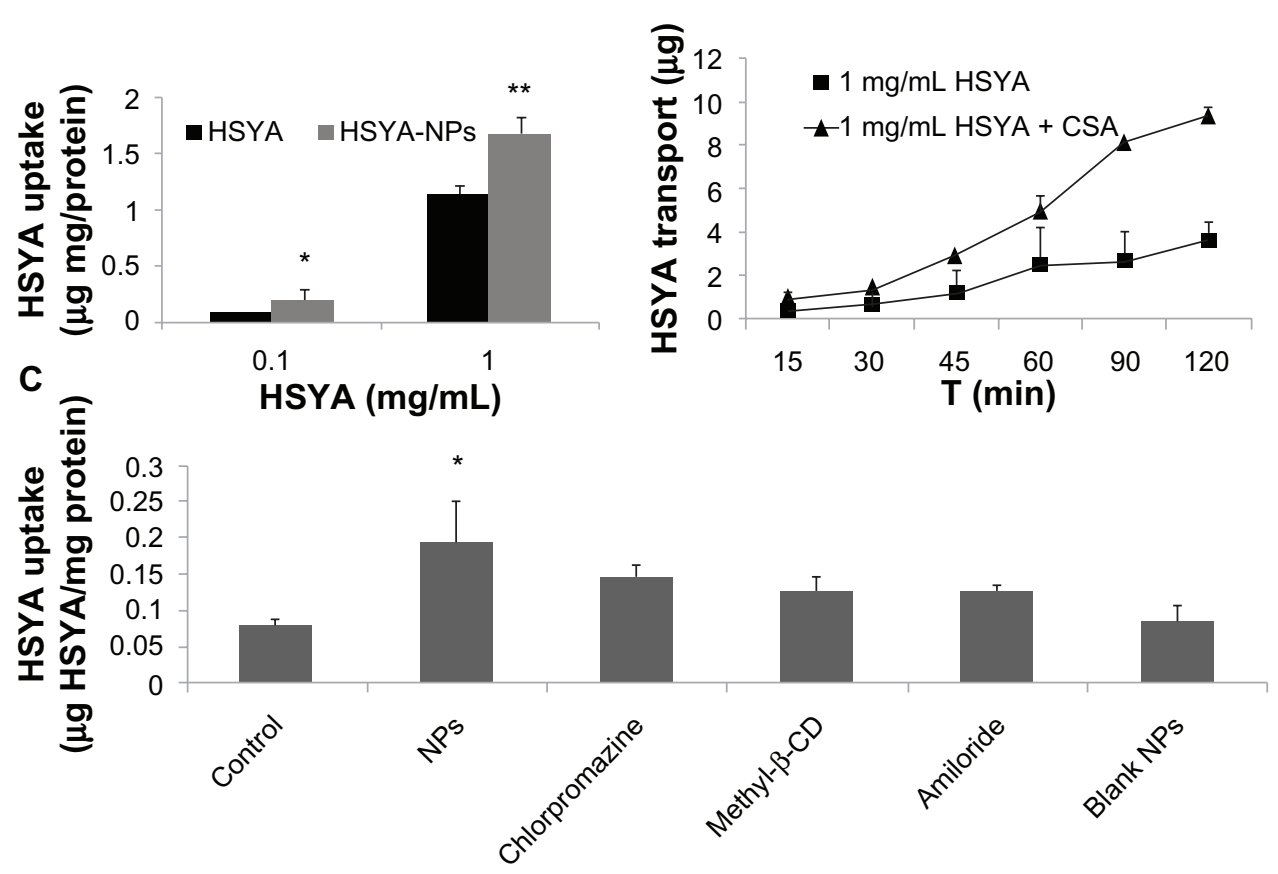

Figure 4 (A) HSYA solution and HSYA nanoparticle uptake by Caco-2 monolayers after incubation for 2 hours; (B) HSYA transport across Caco-2 cells (surface area of monolayer $\left.=1.12 \mathrm{~cm}^{2}\right)$; (C) Endocytotic inhibitor studies on Caco-2 cells of HSYA-NPs.

Notes: ${ }^{*} P<0.05$; $* * P<0.0$ l, compared to control.

Abbreviations: CSA, cyclosporin A; HSYA, hydroxysafflor yellow A; Methyl- $\beta$-CD, methyl- $\beta$-cyclodextrin; NP, nanoparticle; T, time. 


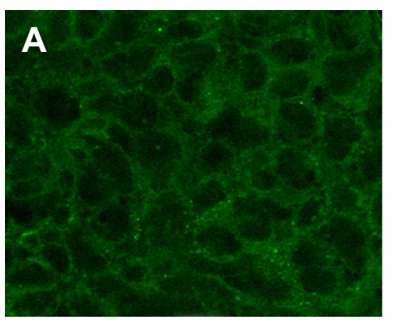

Control

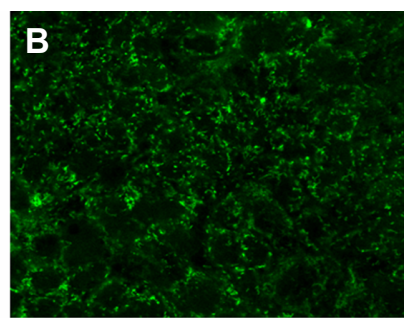

NPs
Figure 5 (A) ZO-I staining in control cell layers not subjected to HSYA nanoparticles; (B) ZO-I staining in cells incubated with I0-fold dilution HSYA-NPs for 2 hours at $37^{\circ} \mathrm{C}$.

Abbreviations: HSYA, hydroxysafflor yellow A; NP, nanoparticle.

ability, ${ }^{1,10}$ which limits its application in clinical practice despite its many prominent pharmacological effects. In our previous studies, ${ }^{11}$ we developed a self-double-emulsifying drug delivery system (SDEDDS), which was composed of water in oil emulsions and hydrophilic surfactants that can self-emulsify into water-in-oil-in-water (w/o/w) double emulsions in the aqueous gastrointestinal environment. This formulation increased the oral bioavailability of HSYA 2.17-fold compared to HSYA solution.
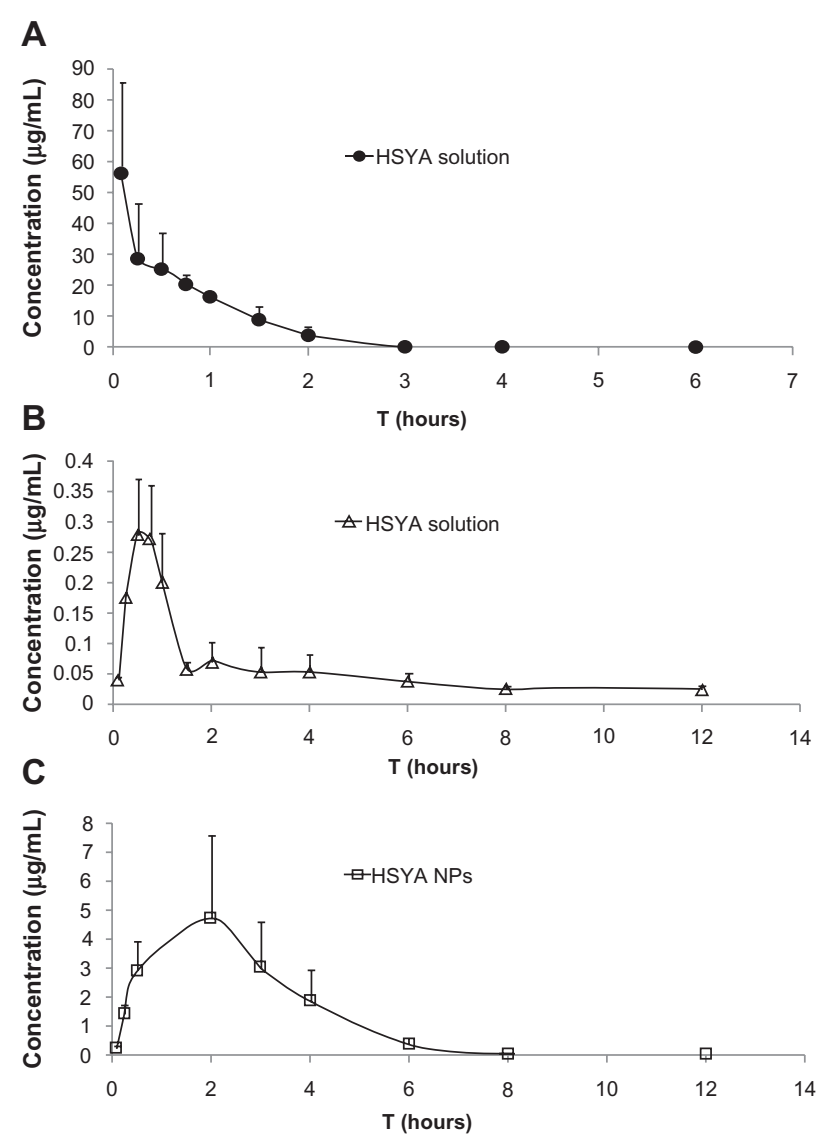

Figure 6 (A) Plasma concentration-time profiles of HSYA after intravenous administration of HSYA solution, (B) oral administration of HSYA solution, and (C) HSYA-NPs to rats at a dose of $25 \mathrm{mg} / \mathrm{kg}$.

Abbreviations: HSYA, hydroxysafflor yellow A; NP, nanoparticle.
Table I Pharmacokinetic parameters after intravenous or oral administration of HSYA formulations to rats

\begin{tabular}{|c|c|c|c|}
\hline & \multirow{2}{*}{$\begin{array}{l}\text { Intravenous } \\
\text { HSYA }\end{array}$} & \multicolumn{2}{|l|}{ Oral } \\
\hline & & $\begin{array}{l}\text { HSYA } \\
\text { solution }\end{array}$ & HSYA-NPs \\
\hline $\mathrm{T}_{\max }(\min )$ & 5 & $45 \pm 15$ & $90.00 \pm 51.96$ \\
\hline $\mathrm{C}_{\max }(\mu \mathrm{g} / \mathrm{mL})$ & $56.51 \pm 29.05$ & $0.33 \pm 0.05$ & $4.80 \pm 2.77^{*}$ \\
\hline $\begin{array}{l}\mathrm{AUC}_{0-12} \\
\text { ( } \mu \mathrm{g} \text { minute/mL) }\end{array}$ & $2,477.68 \pm 221.11$ & $39.92 \pm 6.20$ & $931.53 \pm 406.34 * *$ \\
\hline MRT (minute) & $77.47 \pm 6.74$ & $227.20 \pm 12.29$ & $311.12 \pm 26.52 * *$ \\
\hline $\mathrm{T}_{\text {half }}$ (minute) & $353.32 \pm 64.29$ & $407.6 I \pm 24 I .36$ & $579.97 \pm 216.47$ \\
\hline $\mathrm{F}_{\mathrm{rel}}(\%)$ & - & 100 & 2,333 \\
\hline $\mathrm{F}_{\mathrm{ab}}(\%)$ & 100 & 1.61 & 38 \\
\hline
\end{tabular}

Notes: The values represent mean \pm standard deviation $(n=3) ; * P<0.01 ; * * P<0.05$ compared to control.

Abbreviations: $A \cup C$, areas under the concentration time curve; $C_{\max }$, maximum plasma concentration; $F_{\text {rel }}$, relative bioavailability; $F_{a b}$, absolute bioavailability; HSYA, hydroxysafflor yellow A; MRT, mean retention time; NPs, nanoparticles; $T_{\text {half }}$, halflife; $T_{\text {max }}$, time to maximum plasma concentration.

In this study, we developed a hydrophobic nanoparticle oil solution, the novel formulation of which could be a nanocarrier for hydrophilic drugs with the drug dispersed in the oil phase. Actually, a clear and transparent nanoparticles oil solution was formed when organic solutions A and B were mixed, which means HSYA could be dissolved in organic solvent with soybean phospholipids. After the evaporation of organic solvent, the nanoparticles of HSYA/ soybean phospholipids dispersed in GTCC were formed with the structure of a hydrophilic core and hydrophobic surface. As a water soluble drug, HSYA was wrapped in the core of nanoparticles. No deposition could be observed. The formulation composition and preparation process in the present study was determined after some experimental optimization, as not all kinds of oil and surfactant could form the nanoparticles of "hydrophilic drug dispersed in hydrophobic oil". Due to its pure anhydrous environment, the nanoparticle was more stable for HSYA when compared to SDEDDS. From the pharmacokinetic results (Figure 6 and Table 1), we can see that the nanoparticles increased the absolute bioavailability and relative bioavailability to 23.6- and 23.3-fold, respectively. Figure 1 shows that the nanoparticles exhibited an irregular structure, with a particle size of $49.2 \mathrm{~nm} \pm 5.8 \mathrm{~nm}$. The polydispersity of HSYA nanoparticles was 0.38 , which indicated that the particle size distribution of nanoparticles is relatively non-uniform. In the future, we will optimize the prescription of nanoparticles in order to get uniform size, and to shape rounded particles. In this article, the reversed-dialysis bag method was applied to the in vitro release studies, ${ }^{23}$ and sustained in vitro release was achieved (Figure 2). However, the nanoparticles did not exhibit a sustained release effect in vivo. We speculate 
that there are significant differences between in vivo and in vitro environments.

Results of uptake studies on Caco-2 cells (Figure 4A) showed that the formulation can remarkably increase the membrane permeability of HSYA across Caco-2 cell monolayers at low and high concentrations. However, the endocytotic mechanism of nanoparticles is complex. When cells were pretreated with endocytosis inhibitors, such as chlorpromazine, methyl- $\beta-C D$, and amiloride, for 30 minutes, the uptake of HSYA decreased to some extent, but did not differ significantly (Figure 4C). Based on the endocytosis results, we assume that the uptake process of HSYA nanoparticles may involve a number of factors. As shown from Figure 4B, CsA (a recognized p-glycoprotein inhibitor) could significantly increase the absorption of HSYA on Caco- 2 cells, which indicated that the presence of $\mathrm{p}$ - glycoprotein efflux may be involved in HSYA absorption. Although there are no other studies in the literature discussing the mechanisms for the low absorption of HSYA solution, our preliminary studies support this hypothesis. ${ }^{11}$ Figure 5 shows that the treatment of a monolayer with HSYA nanoparticles at a 1:10 dilution appeared to cause a redistribution of ZO-1. The results indicate that the formulation could break the tight junction of Caco-2 cell layers to promote drug absorption through the paracellular pathway. Meanwhile, the cells were treated with blank-nanoparticles (NPs) for 2 hours followed by another 48-hour incubation with fresh culture medium without blank-NPs, and cell membrane integrity was not influenced as indicated by the TEER results (Figure 3A), suggesting good biocompatibility of the nanoparticles.

\section{Conclusion}

In the present study, a novel oral delivery system of HSYA was developed to improve the oral bioavailability of HSYA. The prepared nanoparticles had a diameter of $49.2 \mathrm{~nm} \pm 5.8 \mathrm{~nm}$ and had sustained release properties. The results of Caco-2 monolayer uptake and pharmacokinetics studies suggest increased HSYA absorption both in vitro and in vivo, which indicates great potential for the future formulation of HSYA.

\section{Acknowledgments}

This study was funded by the Natural Science Foundation of Zhejiang Province, People's Republic of China (No Y2100564; No Y2110124; No Y2100645).

\section{Disclosure}

The authors report no conflicts of interest in this work.

\section{References}

1. Wang S, Sun M, Ping Q. Enhancing effect of Labrafac Lipophile WL 1349 on oral bioavailability of hydroxysafflor yellow A in rats. Int J Pharm. 2008;358(1-2):198-204.

2. Asada H, Douen T, Waki M, et al. Absorption characteristics of chemically modified-insulin derivatives with various fatty acids in the small and large intestine. J Pharm Sci. 1995;84(6):682-687.

3. Dunn SP, Cooney GF, Kulinsky A, et al. Absorption characteristics of a microemulsion formulation of cyclosporine in de novo pediatric liver transplant recipients. Transplantation. 1995;60(12): 1438-1442.

4. Deshmukh DD, Ravis WR, Betageri GV. Improved delivery of cromolyn from oral proliposomal beads. Int J Pharm. 2008;358(1-2): $128-136$.

5. Zang BX, Jin M, Si N, Zhang Y, Wu W, Piao YZ. [Antagonistic effect of hydroxysafflor yellow A on the platelet activating factor receptor]. Yao Xue Xue Bao. 2002;37(9):696-699. Chinese.

6. Zhu H, Wang Z, Ma C, et al. Neuroprotective effects of hydroxysafflor yellow A: in vivo and in vitro studies. Planta Med. 2003;69(5): 429-433.

7. Chu D, Liu W, Huang Z, Liu S, Fu X, Liu K. Pharmacokinetics and excretion of hydroxysafflor yellow A, a potent neuroprotective agent from safflower, in rats and dogs. Planta Med. 2006;72(5):418-423.

8. Qin F, Huang X. Guanxin II (II) for the management of coronary heart disease. Chin J Integr Med. 2009;15(6):472-476.

9. Liu SX, Zhang Y, Wang YF, et al. Upregulation of heme oxygenase-1 expression by hydroxysafflor yellow A conferring protection from anoxia/reoxygenation-induced apoptosis in $\mathrm{H} 9 \mathrm{c} 2$ cardiomyocytes. Int J Cardiol. 2012;160(2):95-101.

10. Qi J, Zhuang J, Wu W, et al. Enhanced effect and mechanism of waterin-oil microemulsion as an oral delivery system of hydroxysafflor yellow A. Int J Nanomedicine. 2011;6:985-991.

11. Lv LZ, Tong CQ, Lv Q, et al. Enhanced absorption of hydroxysafflor yellow A using a self-double-emulsifying drug delivery system: in vitro and in vivo studies. Int J Nanomedicine. 2012;7:4099-4107.

12. Hu W, Dong H, Li YZ, Hu XT, Han GJ, Qu YB. A high-throughput model for screening anti-tumor agents capable of promoting polymerization of tubulin in vitro. Acta Pharmacol Sin. 2004;25(6): 775-782.

13. Boukhettala N, Claeyssens S, Bensifi M, et al. Effects of essential amino acids or glutamine deprivation on intestinal permeability and protein synthesis in HCT-8 cells: involvement of GCN2 and mTOR pathways. Amino Acids. 2012;42(1):375-383.

14. Ma TY, Nguyen D, Bui V, Nguyen H, Hoa N. Ethanol modulation of intestinal epithelial tight junction barrier. Am J Physiol. 1999; 276(4 Pt 1):G965-G974.

15. Das S, Ng WK, Kanaujia P, Kim S, Tan RB. Formulation design, preparation and physicochemical characterizations of solid lipid nanoparticles containing a hydrophobic drug: effects of process variables. Colloids Surf B Biointerfaces. 2011;88(1):483-489.

16. $\mathrm{Hu} \mathrm{K}, \mathrm{Cao} \mathrm{S}, \mathrm{Hu}$ F, Feng J. Enhanced oral bioavailability of docetaxel by lecithin nanoparticles: preparation, in vitro, and in vivo evaluation. Int J Nanomedicine. 2012;7:3537-3545.

17. Roger E, Kalscheuer S, Kirtane A, et al. Folic acid functionalized nanoparticles for enhanced oral drug delivery. Pharmaceutics. 2012;9(7):2103-2110.

18. Tian Y, Yang ZF, Li Y, et al. Pharmacokinetic comparisons of hydroxysafflower yellow A in normal and blood stasis syndrome rats. J Ethnopharmacol. 2010;129(1):1-4.

19. Song L, Zhu Y, Jin M, Zang B. Hydroxysafflor yellow a inhibits lipopolysaccharide-induced inflammatory signal transduction in human alveolar epithelial A549 cells. Fitoterapia. 2013;84:107-114.

20. Sun L, Yang L, Xu YW, et al. Neuroprotection of hydroxysafflor yellow $\mathrm{A}$ in the transient focal ischemia: inhibition of protein oxidation/ nitration, 12/15-lipoxygenase and blood-brain barrier disruption. Brain Res. 2012;1473:227-235. 
21. Zhu HB, Zhang L, Wang ZH, et al. Therapeutic effects of hydroxysafflor yellow A on focal cerebral ischemic injury in rats and its primary mechanisms. J Asian Nat Prod Res. 2005;7(4):607-613.

22. Liu L, Duan JA, Tang Y, et al. Taoren-Honghua herb pair and its main components promoting blood circulation through influencing on hemorheology, plasma coagulation and platelet aggregation. J Ethnopharmacol. 2012;139(2):381-387.
23. Levy MY, Benita S. Drug release from submicronized o/w emulsion - a new in vitro kinetic evaluation model. Int J Pharm. 1990;66(1-3): 29-37.

International Journal of Nanomedicine

\section{Publish your work in this journal}

The International Journal of Nanomedicine is an international, peerreviewed journal focusing on the application of nanotechnology in diagnostics, therapeutics, and drug delivery systems throughout the biomedical field. This journal is indexed on PubMed Central, MedLine, CAS, SciSearch $\AA$, Current Contents ${ } /$ Clinical Medicine,
Journal Citation Reports/Science Edition, EMBase, Scopus and the Elsevier Bibliographic databases. The manuscript management system is completely online and includes a very quick and fair peer-review system, which is all easy to use. Visit http://www.dovepress.com/ testimonials.php to read real quotes from published authors.

Submit your manuscript here: http://www.dovepress.com/international-journal-of-nanomedicine-journal 